\title{
Moisture Content of Composites Under Transient Conditions
}

\author{
GEORgE S. SPRINGER \\ Department of Mechanical Engineering \\ The University of Michigan \\ Ann Arbor, Michigan 48109 \\ (Received 1)ecember 27, 1976)

\begin{abstract}
The moisture content and moisture distribution of a fiber reinforced composite were calculated under conditions simulating the typical, 20 year service life of an aircraft. The results indicate that the complete transient analysis of the problem must be performed in order to establish a) the long term moisture effects, b) the conditions for accelerated testing, c) the "worst case" design conditions, and d) methods to interpret field monitoring programs on moisturing gain.
\end{abstract}

\section{SCOPE}

ONE OF THE KEY questions in using composite materials as aircraft components is the degradation of the material due to moisture absorption through long years of service. The objective of this investigation was to calculate the moisture distribution and the moisture content of a composite material exposed to air with varying temperature and varying relative humidity. The temperature and moisture histories included conditions simulating runway storage and supersonic flight through 20 years of service. The results obtained provide the moisture content, the moisture distribution, and an evaluation of the feasibility of introducing moisture in the laboratory in a manner that would simulate the real life moisture characteristics of the material.

\section{CONCLUSIONS}

The following problem was investigated. An uncoated or coated plate, made of a fiber reinforced composite material, is exposed on both sides to humid air (Figure 1). The temperature and the relative humidity of the air vary with time in a cyclic manner. Calculations were performed to determine the moisture content (percent weight gain) of the material as a function of time, and the moisture distribution inside the material as a function of position and time. The calculations yield the following general conclusions. 


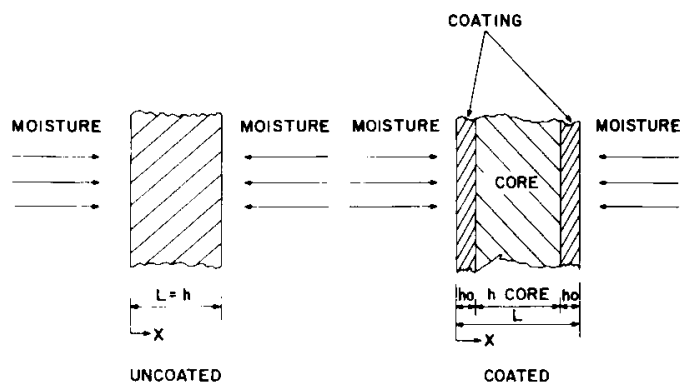

Figure 1. Geometry of the problem.

\section{Transient Ambient Conditions}

a) After 10 years of exposure to the actual (transient) environment the moisture content nearly reaches a "steady state". Afterwards, the moisture content fluctuates only slightly about this steady state value.

b) The moisture distribution inside the material never attains a steady state. The moisture distribution changes continuously, the moisture concentration depending upon time and position. After about 6 years most of the changes in the concentration occur within a narrow layer ("boundary layer") near the exposed surface.

\section{Constant Ambient Conditions}

a) The actual variation of the moisture content and the moisture distribution with time cannot be duplicated in accelerated tests by simply replacing the transient ambient conditions by a constant temperature and a constant relative humidity.

b) The "steady state" moisture distribution inside the material (but outside the boundary layer), and the "steady state" moisture content (attained after 10-20 years exposure to the actual environment) can be approximated by constant ambient conditions. However, the appropriate constant relative humidity to be used in the simulation cannot be guessed a priori, but must be determined by solving the entire transient problem.

\section{The Effects of Temperature and Humidity}

The ambient temperature and humidity affect significantly both the moisture content and the moisture distribution. Although the results presented apply only to the conditions of this study, the general trends indicated by the results are expected to be valid for other conditions. However, for each new problem the complete transient analysis must be performed in order to evaluate the actual moisture content, the actual moisture distribution, and the ambient conditions resulting in the "worst case" design conditions. 


\section{Coated Composites}

The conclusions stated in points 1-3 apply to both uncoated and coated composites. For a coated composite the coating may also reduce significantly the moisture content of the core even if the coating is to some extent permeable to moisture.

\section{Moisture Gain Monitoring}

Field or laboratory monitoring of moisture gain can be interpreted with confidence if it is supplemented with a transient analysis. The measured weight gain should be checked against that predicted.

a) Since steady-state boundary conditions cannot make this prediction, a transient analysis is required. Any anomoly between the measured and the predicted weight gains may indicate that processes other than diffusion is operating. Water may have penetrated through cracks and lodged in voids, joints and honeycomb.

b) Since there is no available technique to measure the distribution of moisture across the thickness and from point to point in a laminate, a transient analysis can be used to determine the moisture distribution. From the predicted distribution, the stress distribution that may be damaging to the structure may also be assessed. It is believed that a meaningful monitoring program will require an accompanying transient analysis.

\section{PARAMETERS USED IN THE CALCULATIONS}

The calculations were performed for a transient environment simulating the 20 year service life of an aircraft. The atmospheric variations of temperature and relative humidity are shown in Figure 2 . The temperature and humidity were assumed to vary in a cyclic manner, each cycle repeating every 6 days. Two different cycles were considered: a "high humidity" cycle with the relative humidity kept constant at $82 \%$, except during flight when the humidity was 0 percent, and a "low humidity" cycle where the relative humidity of the environment was reduced as the temperature increased. The relative humidity was calculated from the relationship

$$
H=\frac{P_{v}}{P_{g}}
$$

where $P_{v}$ is the partial pressure of the vapor as it exists in the mixture, and $P_{g}$ is the saturation pressure of the vapor at the same temperature. The moisture content (and hence $P_{v}$ ) was taken to be constant. Accordingly, the relative humidity at temperature $T$ was related to the saturation pressure at $72 \mathrm{~F}$ and relative humidity at $82 \%$ by the expression

$$
(H)_{T}=\frac{P_{v}}{\left(P_{g}\right)_{T}}=\frac{\left(P_{g}\right)_{72 F}}{\left(P_{g}\right)_{T}}(82)
$$



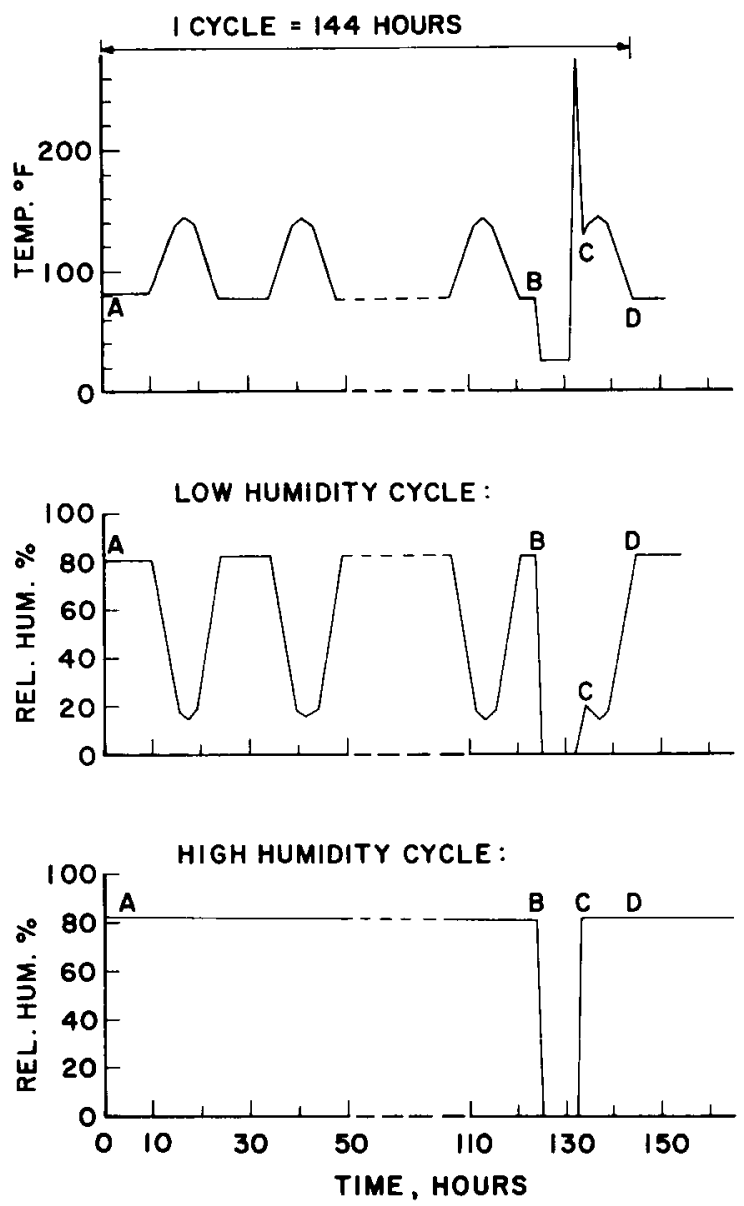

Figure 2. Ambient temperature and humidity used in the calculations.

In practice, the ambient conditions follow more closely the "low humidity" cycle than the high humidity one. Therefore, most of the results were generated for the low humidity cycle. Selected results were obtained for the high humidity cycle. Results were also generated for various constant temperature, constant humidity ambients.

The material properties and the geometries used in the calculations are summarized in Table 1. 
Table 1. Properties of Materials Used in the Calculations.

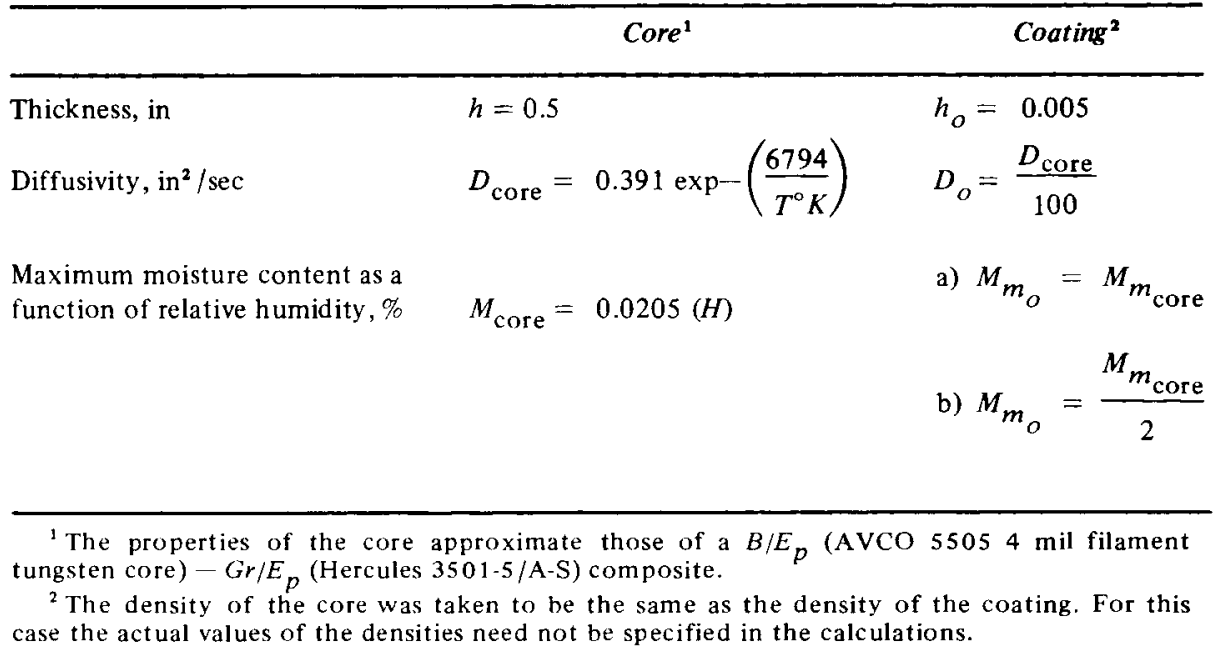

\section{RESULTS}

Moisture contents and moisture distributions were calculated using the computer code "W8GAIN" developed in the Mechanical Engineering Department, The University of Michigan. The analysis forming the basis of this code is described in the Appendix.

The moisture content and moisture distribution as a function of time for an uncoated composite are given in Figures 3 and 4. For the actual ambient conditions results were plotted for two extreme situations, namely before flight (point $B$, Figure 2) and after flight (point C, Figure 2).

It is seen from Figure 3 that the moisture content nearly reaches a "steady state" value after about 10 years. However, even after 10 years there is a small variation in the moisture content as the material absorbs moisture during runway storage and dries during flight.

The "steady state" moisture content depends strongly on the actual ambient conditions. The "steady state" moisture content cannot be guessed from the ambient conditions alone, but must be determined from the complete transient analysis. Conversely, the transient ambient conditions which would yield a given moisture content cannot be obtained without the transient analysis. In other words, the transient ambient conditions resulting in a prescribed (e.g. "worst case") moisture content cannot be established without first solving the transient problem.

The steady state moisture content reached under transient ambient conditions can be approximated by employing constant ambient conditions. The constant temperature and humidity appropriate for this approximation must be determined 


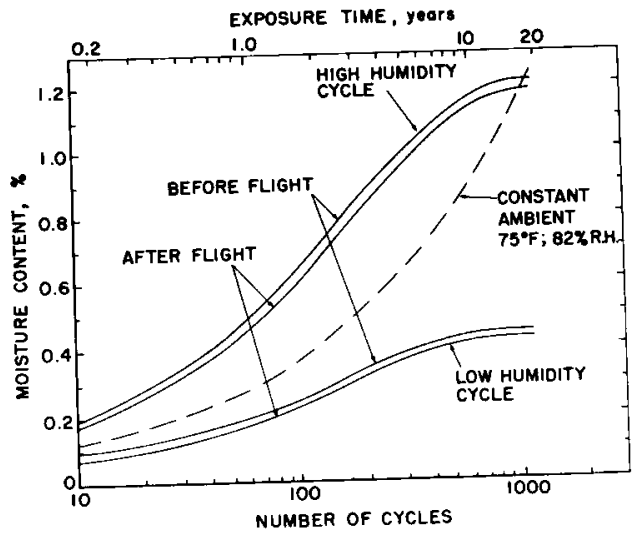

Figure 3. The variation of moisture content with time. Uncoated composite with properties specified in Table 1. Actual ambient conditions given in Figure 2.

ACTUAL AMBIENT - BEFORE FLIGH .... AFTER FLIGHT

---. CONSTANT AMBIENT

$75 F, 82 \% R H$
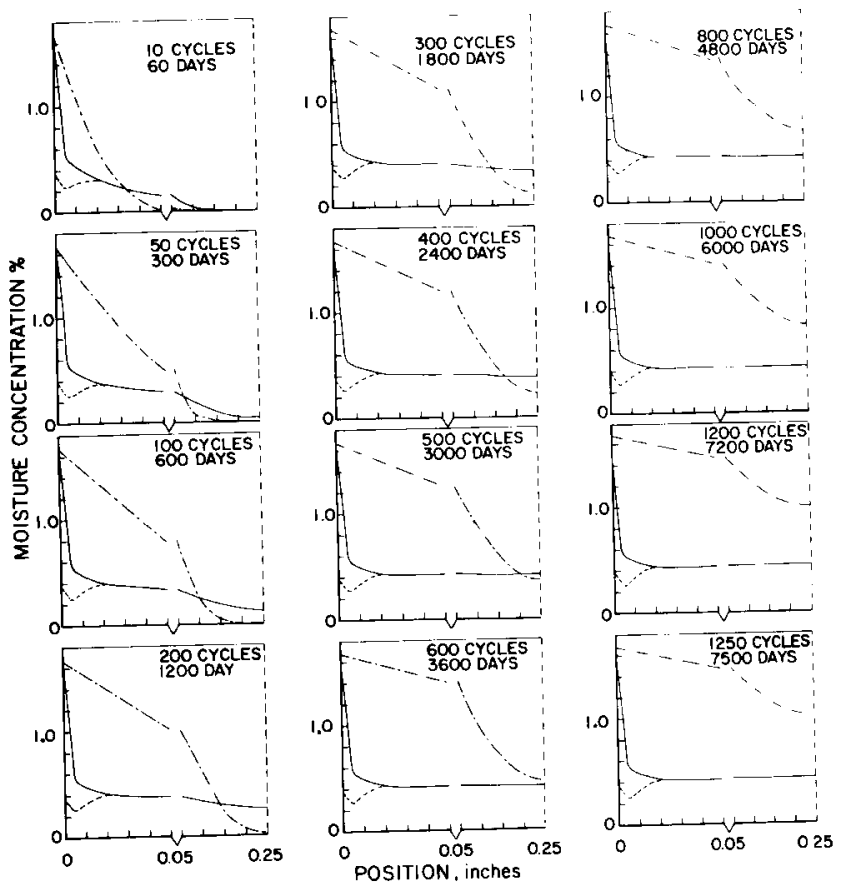

Figure 4. The variation of the moisture distribution with time. Uncoated composite with properties specified in Table 1. Actual ambient condition is for low humidity cycle given in Figure 2. (Note change in horizontal scale) 
by matching the moisture contents given by the transient and by the constant ambient conditions. This procedure again necessitates the solution of the transient problem.

The variation of moisture content with time for different constant temperatures is illustrated in Figure 5. The ambient relative humidity used in these simulations was set at $21.5 \%$, a value which yields the $0.44 \%$ steady state moisture content given by the actual low humidity cycle.

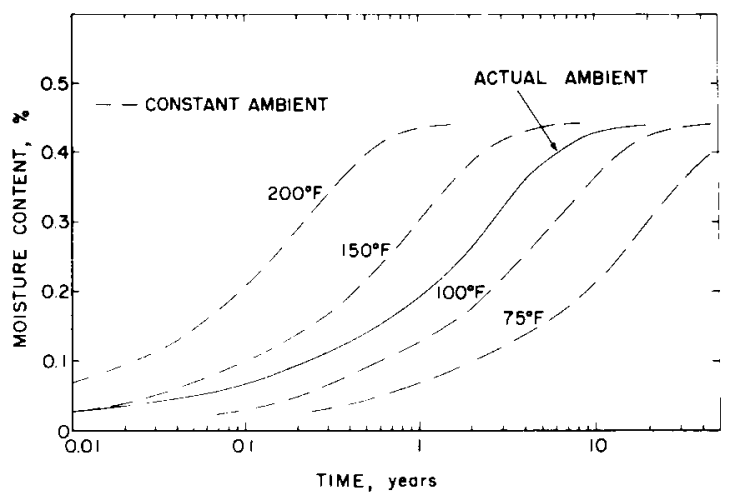

Figure 5. The variation of moisture content with time. Uncoated composite with properties specified in Table 1. Actual ambient condition is for the low humidity clcle given in Figure 2. Constant ambient conditions are for $21.5 \%$ relative humidit", and for the different temperatures indicated.

It is inportant to note that the speed by which moisture penetrates the material (i.e. the time required to reach steady state) is insensitive to the relative humidity and is governed largely by the temperature. The time needed to reach 90 to 99.9 percent of the steady state moisture content at different constant ambient temperatures is illustrated in Figure 6. At $150 \mathrm{~F}$ it takes about seven years to attain the steady state moisture content. At $200 \mathrm{~F}$ the time required is only $\sim 1.5$ years. A change in the relative humidity would not influence appreciably the time required to reach steady state but would affect significantly the moisture content at steady state. This is demonstrated in Figure 3 where moisture contents are presented for both the low humidity and high humidity cycles. The time to reach steady state is nearly the same for both cycles. However, the steady state moisture content almost triples from the low to the high humidity cycle.

The moisture distribution never reaches a steady state but varies continuously (Figure 4). Nevertheless, after about 6 years most of the variations occur within a narrow layer near the exposed surface. This layer is denoted here as the "boundary 


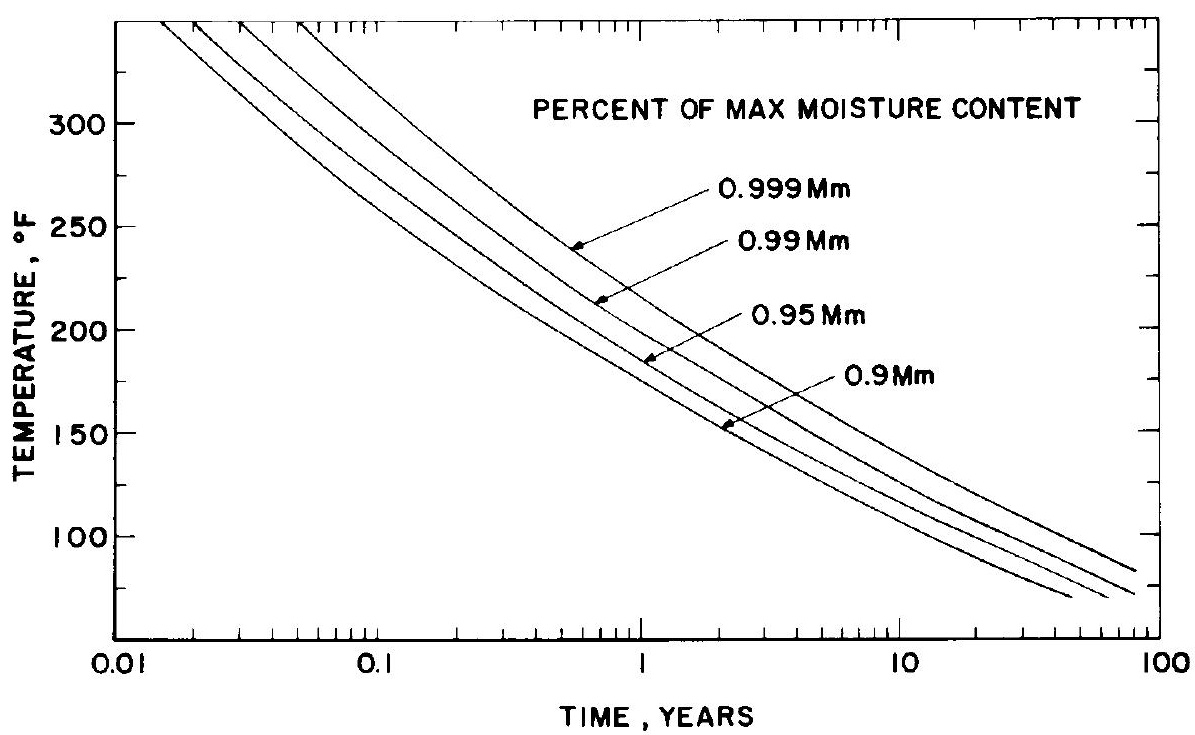

Figure 6. The time required to reach 90 to $99.9 \%$ of the actual steady state moisture content at different constant ambient temperatures. Uncoated composite with properties specified in Table 1.

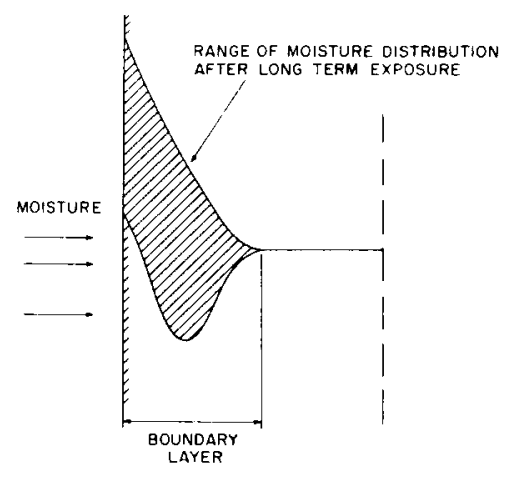

Figure 7. Schematic of the fluctuation of the moisture concentration during each ambient cycle after long time exposure to the moist environment. layer" (Figure 7). Outside of the boundary layer the distribution becomes reasonably steady after 6 years.

Owing to the continuous variation of the moisture distribution, the distribution resulting from a transient ambient cannot be simulated accurately by a constant environment. The steady state distribution outside the boundary layer can be approximated using constant temperature, constant humidity air. The appropriate constant conditions must be carefully selected on the basis of the results of the transient analysis. Seemingly logical temperature and humidity may result in completely erroneous moisture distribution and moisture content. This is illustrated in Figures 3 and 4 where the moisture contents and the moisture distributions calculated for runway conditions $(72 \mathrm{~F}, 82 \%$ rel. hum.) are compared to the actual results. The large differences between the actual and constant ambient results 
attest to the difficulty of selecting arbitrarily the constant ambient conditions.

The foregoing results indicate that it is possible to introduce moisture in the laboratory in a manner that would approximate the long term real life moisture characteristics of the material. However, it is imperative that the constant ambient conditions used in simulating the actual ambient be selected correctly. The following procedure must be employed in determining the constant ambient conditions which, in a reduced time, provide the long term, steady state moisture content and moisture distribution.

1) Using the transient analysis calculate the variation of the moisture content and moisture distribution with respect to time under the actual ambient conditions.

2) From the results of the transient analysis determine the steady state moisture content $M_{s s}$ and the steady state moisture distribution outside the boundary layer.

3) Using the empirical relationship (see Appendix, Equation A15)

$$
H=\left(\frac{M_{s s}}{100 a}\right)^{1 / b}
$$

determine the constant relative humidity $H$ which yields the steady state moisture content $M_{s s}$.

4) For an initially dry material the time required to reach 99.9 percent of the steady state moisture content is [1],

$$
t_{s s}=\frac{0.67 D_{x}}{h^{2}}
$$

The mass diffusivity $D_{x}$ is related to the temperature $T$ by the Arrhenius equation

$$
D_{x}=d \exp \left(-\frac{f}{T}\right)
$$

where $d$ and $f$ are constants which depend on the material. The temperature $T$ is in degree Rankine. Equations (4) and (5) yield the following expression for the constant temperature needed to reach the steady state moisture content in the prescribed time $t_{s s}$

$$
T=-\frac{f}{\operatorname{In}\left(h^{2} t_{s s} / 067 d\right)}
$$


Equation (6) can also be used, of course, to estimate the time required to reach steady state for a given ambient temperature.

Note that Equation (6) is valid only if the material is dry initially. For nonuniform initial moisture distribution (which is usually the case when the material is not dry) $T$ (or $t_{s s}$ ) must be calculated numerically.

The foregoing results are for uncoated composites. Calculations were also performed for a composite covered with a thin layer of coating to evaluate the effects of the coating. The parameters which influence the protection offered by the coating are a) the mass diffusivity of the coating, b) the maximum moisture content of the coating, c) density of the coating, and d) the coat thickness. In obtaining the following results these parameters were set at the values given in Table 1 .

The moisture content and the moisture distributions as a function of time for the coated composite are presented in Figures 8-10. Results are given for the two cases when the maximum moisture content of the coating is a) the same and b) half of that of the core. All other relevant parameters (diffusivity, density, thickness) were unchanged.

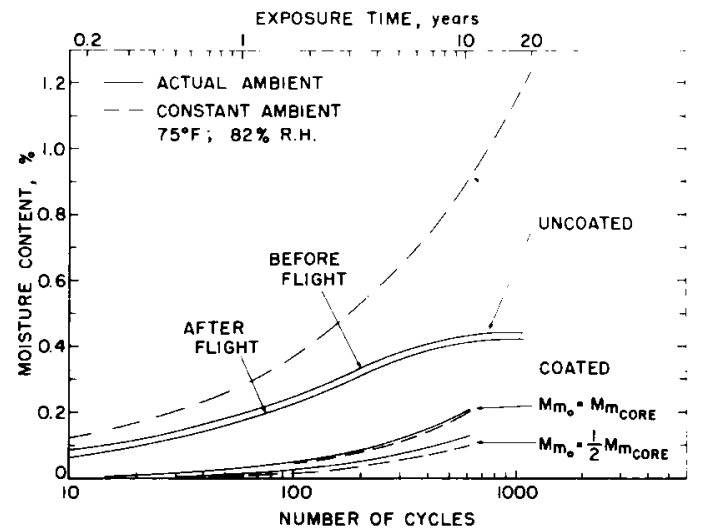

Figure 8. The variation of moisture content with time. Coated and uncoated composites with properties specified in Table 1. The results for the coated composites are for the low humidity cycle given in Figure 2.

The results for the coated material show the same trends as the results for uncoated material. Thus the conclusions enumerated previously for the uncoated composites also apply to coated materials. One point needs noting. The constant temperature constant humidity ambient seemingly approximates the moisture content given by the actual ambient. This result, however, is fortuitous. It is due to the fact that the moisture concentration given by the constant ambient under- 
ACTUAL AMBIENT

— BEFORE FLIGHT AFTER FLIGHT
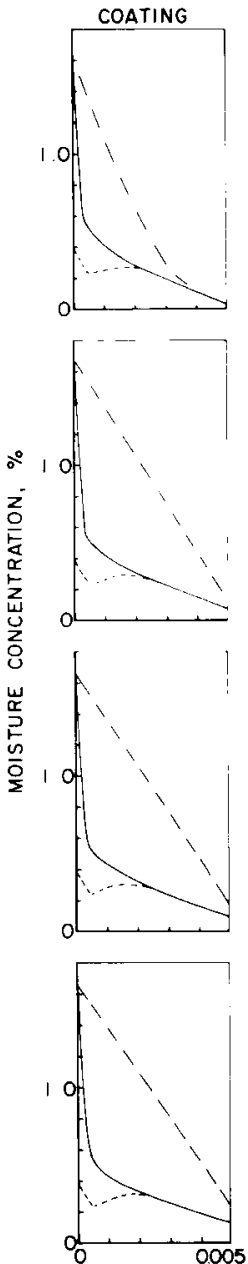

0005
50 CYCLES

300 DAYS

CORE

10 CYCLES

60 DAYS
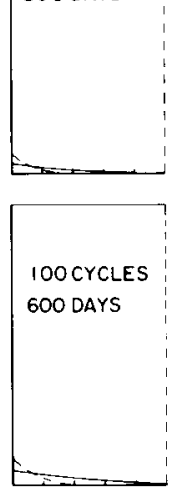

200 CYCLES

IZOODAYS

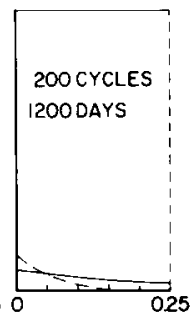

POSITION, inches

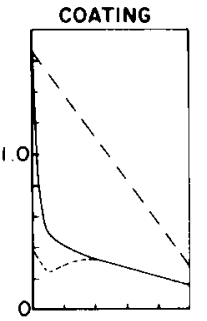

CORE
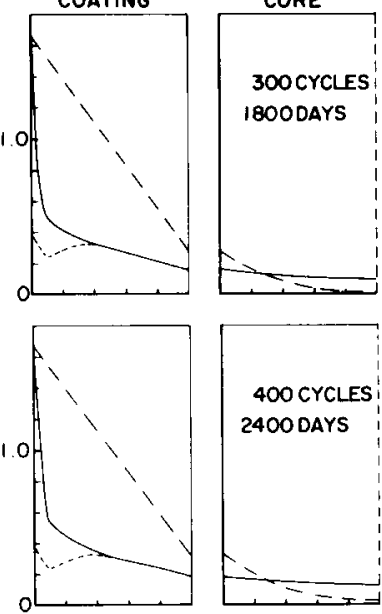

400 CYCLES

2400 DAYS
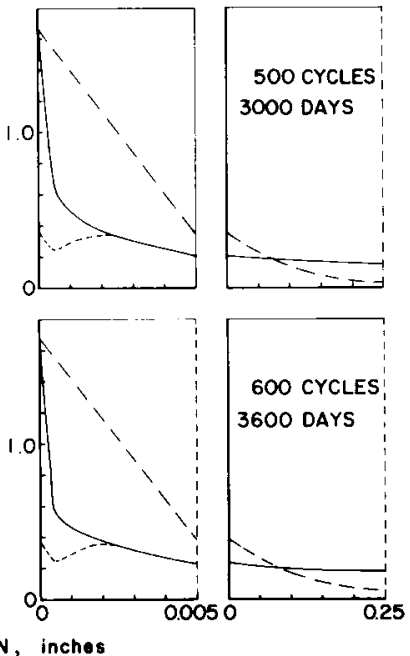

Figure 9. The variation of moisture distribution with time. Coated composite with $M_{m_{0}}=M_{m_{\text {core }}}$ Material properties specified in Table 1. Actual ambient condition is for the low humidity cycle given in Figure 2. (Note change in horizontal scale) 


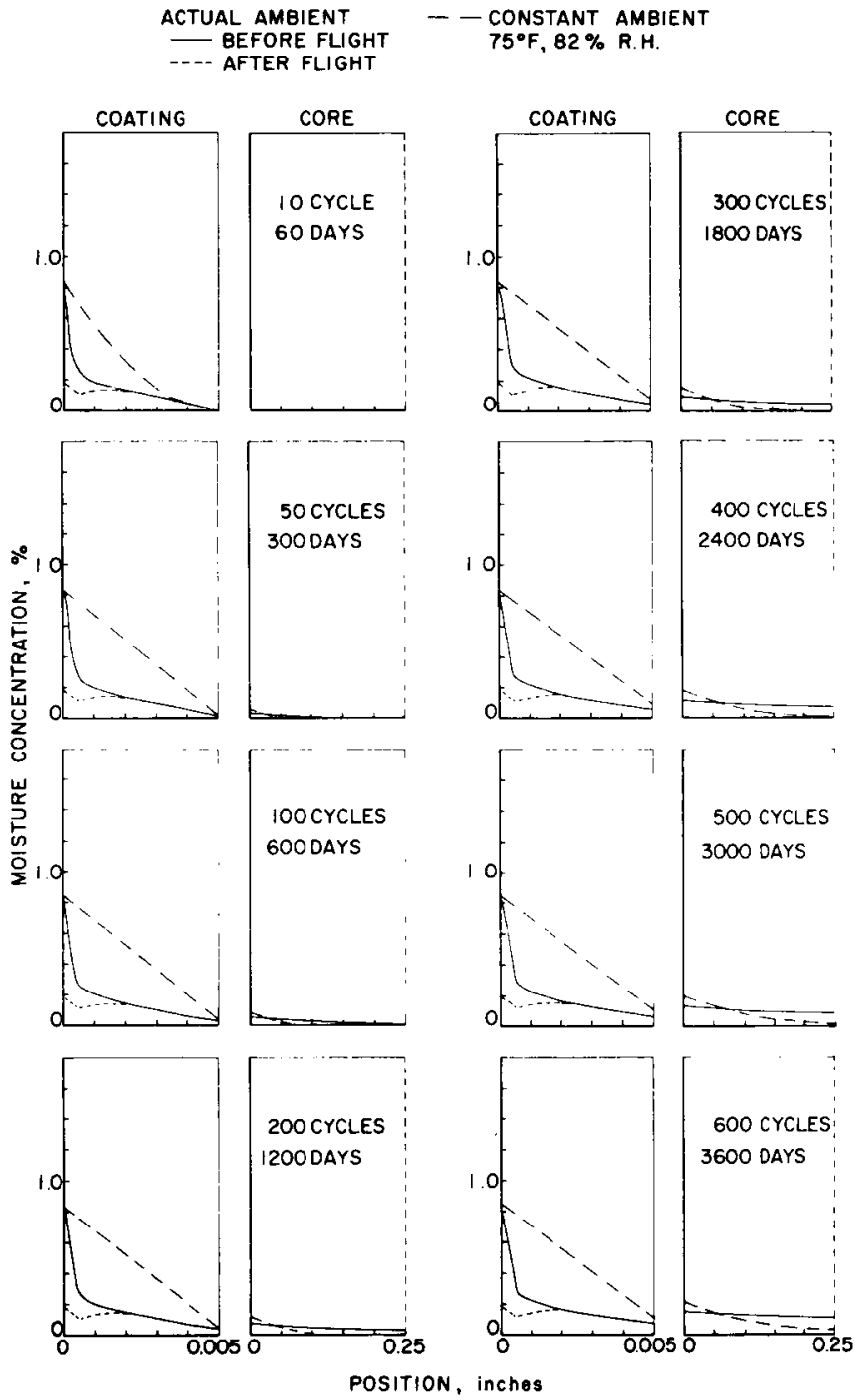

Figure 10. The variation of moisture distribution with time. Coated composite with $M_{m_{o}}=1 / 2 M_{m_{\text {core }}}$ Material properties specified in Table 1. Actual ambient condition is for the low humidity cycle given in Figure 2. (Note change in horizontal scale) 
estimates the actual moisture concentration in the coating and overestimates it inside the core (Figures 9 and 10). These two effects tend to cancel each other in the problem investigated here.

As is seen from Figures 8-10 the coating reduces the amount of moisture in the core significantly even though the coat is permeable to moisture. In this study the diffusivity of the coating was $1 / 100$ of that of the core. A coating material with such a diffusivity reduces the moisture content by factors of two to four, the reduction being larger with lower values of $M_{m}$ for the coating.

\section{APPENDIX}

Analysis

Consider a plate of thickness $L$ exposed on two sides to the same humid air (Figure 1). The plate is taken to be one dimensional so that the moisture concentration and temperature inside the plate vary only in the $x$ direction. It is further assumed that a) at any instant the temperature inside the material is the same as the ambient temperature, b) the mass diffusivity of the material $D_{x}$ depends only on the temperature, but is independent of the moisture content. With these assumptions the moisture concentration $c$ is described by the Fick equation [1]

$$
\frac{\partial c}{\partial t}=D_{x} \frac{\partial^{2} c}{\partial x^{2}}
$$

Equation (A1) must be solved together with the appropriate initial and boundary conditions. Initially (at time $t \leqq 0$ ) the moisture concentration at each point inside the material must be specified

$$
c=c_{i}(x) \quad 0 \leqslant x \leqslant L ; \quad t \leqslant 0
$$

At later times $(t>0)$ the temperature $T_{a}$ and the relative humidity $H$ of the ambient air as a function of time must be given

$$
\begin{aligned}
& T_{a}=T_{a}(t) \\
& H=H(t)
\end{aligned} \quad x=o ; \quad x=L ; \quad t>o
$$

As noted above the temperature inside the material is taken to be the same as the ambient temperature, i.e.

$$
T=T_{a}(t) \quad o \leqslant x \leqslant L ; \quad t>o
$$


The moisture concentration at the surface of the material, denoted by $c_{m}$, is related to the relative humidity by the following empirical relationship [1]

$$
c_{m}=\rho_{d} a H^{b}
$$

where $\rho_{d}$ is the density of the dry material; $a$ and $b$ are empirical constants which depend on the material.

For a coated material the following two conditions are to be satisfied at the interface between the coating and the core.

1) The mass of the moisture crossing the surfaces of the coating and the core per unit area and unit time are equal

$$
\left.D_{x} \frac{\partial c}{\partial x}\right|_{0}=\left.D_{x} \frac{\partial c}{\partial x}\right|_{\text {core }} \quad \text { at interface }
$$

The subscript $O$ refers to the coating.

2) The moisture concentrations at the surface of the coating and at the surface of the core correspond to the same relative humidity

$$
c_{m_{0}} \longrightarrow H \leftarrow c_{m_{\text {core }}} \quad \text { at interface }
$$

or, using Equation (A5)

$$
\left(\sqrt[b]{\frac{c_{m}}{a \rho_{d}}}\right)_{o}=\left(\sqrt[b]{\frac{c_{m}}{a \rho_{d}}}\right)_{\text {core }} \text { at interface }
$$

Equations (A1)-(A8) specify the problem. The solution to these equations provide the moisture concentration $c$ as a function of position and time. In addition, we are interested in the percent moisture content, as manifested by the mass (weight) gain of the material. The percent moisture content is defined as

$$
M=\frac{\text { mass of moist material }- \text { mass of dry material }}{\text { mass of dry material }}(100)=\frac{W-W_{d}}{W_{d}}(100)
$$

The total mass of the moisture in the material $W_{c}$ is obtained by integrating the moisture concentration over the thickness

$$
w_{c}=A \int_{o}^{L} c d x
$$


$A$ is the surface area. The total mass of the moist material is

$$
w=w_{d}+w_{c}
$$

and thus Equation (A9) may be rearranged in the form

$$
M=\frac{W_{c}}{W_{d}}(100)
$$

The maximum moisture content denoted by the subscript $m$, is

$$
M_{m}=\frac{\left(W_{c}\right)_{m}}{W_{d}}(100)=\frac{c_{m} A L}{\rho_{d} A L}(100)=\frac{c_{m}}{\rho_{d}}(100)
$$

Using Equations (A10), (A12) and (A13) the moisture content may be expressed as

$$
M=\frac{W_{c}}{\left(W_{c}\right)_{m}} M_{m}(100)=\frac{W_{c}}{c_{m} L} M_{m}(100)
$$

The percent moisture content as a function of time can be calculated from Equation (AI4).

Equations (A5) and (A15) give the relationship between the maximum moisture content and the ambient relative humidity

$$
M_{m}=a(H)^{b}(100)
$$

It is advantageous to present the moisture concentration in terms of the dimensionless parameter

$$
c^{*}=\frac{\left(c+\rho_{d}\right)-\rho_{d}}{\rho_{d}}(100)=\frac{c}{\rho_{d}}(100)
$$

Figures $3-10$ represent numerical solutions to the above set of equations.

\section{ACKNOWLEDGMENTS}

This work was supported by the Air Force Materials Laboratory, WrightPatterson Air Force Base, Dayton, Ohio. The author thanks Mr. H. Bina for his help with the numerical calculations. 


\section{NOMENCLATURE}

$a, b$ empirical constants, see Equation (A15) (dimensionless)

$A$ area $\left(\right.$ in $\left.^{2}\right)$

c moisture concentration inside the material $\left(\mathrm{g} / \mathrm{in}^{3}\right)$

$c^{*} \quad$ dimensionless moisture concentration defined by Equation (A16)

$D_{x}$ mass diffusivity in $x$ direction $\left(\mathrm{in}^{2} / s\right)$

$d \quad$ constant used in the Arrhenius equation, see Equation (5) $\left(\mathrm{in}^{2} / \mathrm{s}\right)$

$f$ constant used in the Arrhenius equation, see Equation (5) $\left({ }^{\circ} R\right)$

$h$ thickness of material (in)

$H$ relative humidity of ambient (percent)

$L \quad$ total thickness of material, see Figure 1 (in)

$M$ moisture content, see Equation (A9) (percent)

$M_{m}$ maximum moisture content (percent)

$P_{v} \quad$ partial pressure of vapor $\left(\mathrm{lbf} / \mathrm{in}^{2}\right)$

$P_{g} \quad$ saturation pressure of vapor $\left(\mathrm{lbf} / \mathrm{in}^{2}\right)$

$t$ time (s)

$T$ temperature $(F$ or $R)$

$W$ total mass of moist material (lbm)

$W_{c}$ mass of moisture (lbm)

$W_{d}$ mass of dry material (lbm)

$x \quad$ coordinate direction, see Figure 1 (in)

$\rho_{d} \quad$ density of dry material $\left(\mathrm{lbm} / \mathrm{in}^{3}\right)$

\section{Subscripts}

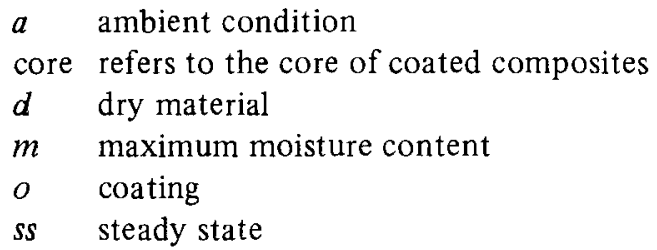

\section{REFERENCE}

1. C. H. Shen and G. S. Springer, "Moisture Absorption and Desorption of Composite Materials," J. Composite Materials, Vol. 10 (1976), p. 2. 


\section{COMPOSITE MATERIALS WORKSHOP}

A five-day "Composite Materials Computation Workshop" to provide users and producers of composites with a practical guide to solving problems in design and testing will be held March 28 to April 1, 1977, at the University of California at Berkeley.

The workshop co-chairmen and principal instructors will be Stephen W. Tsai, of the Air Force Materials Laboratory at Wright-Patterson AFB; Edward M. Wu, of the University's Lawrence Livermore Laboratory; and H. Thomas Hahn, of the University of Dayton Research Institute.

Lectures, practice sessions and drills will be devoted to demonstrating advanced methods that can be applied to everyday problem solving. Whenever possible, available methods will be condensed to an easily used formula or chart, or to a format that can be implemented readily on a programmable pocket calculator. Participants will be provided with calculators for use during the workshop, and also will be given preprogrammed magnetic cards containing pertinent formulas; the cards may be retained for later use.

The workshop fee is $\$ 475$ and advance registration is required, as enrollment is limited. For program details write to Continuing Education in Engineering, University of California Extension, 2223 Fulton St., Berkely, CA 94720, or call (415) $642-4151$. 